Article

\title{
Conjugate Addition of Indoles to $\alpha, \beta$-Unsaturated Ketones Using a Brønsted Acid Ionic Liquid as an Efficient Catalyst
}

\section{Chuan-Ji Yu and Chen-Jiang Liu *}

School of Chemistry and Chemical Engineering, Xinjiang University, Key Laboratory of Oil and Gas Fine Chemicals, Ministry of Education, 830046 Urumqi, China; E-mail: cjxyycj@126.com (C.-J.Y.)

* Author to whom correspondence should be addressed; E-mail: pxylcj@126.com;

Tel.: +86-9918581211; Fax: +86-9918582809.

Received: 6 July 2009; in revised form: 20 August 2009 / Accepted: 24 August 2009 /

Published: 27 August 2009

\begin{abstract}
The Brønsted acid ionic liquid $\left[\mathrm{PyN}\left(\mathrm{CH}_{2}\right)_{4} \mathrm{SO}_{3} \mathrm{H}\right]\left[p-\mathrm{CH}_{3} \mathrm{PhSO}_{3}\right]$ has been reported as an efficient catalyst for the Michael addition reaction of indoles to $\alpha, \beta$ unsaturated ketones. Satisfactory results were obtained, with excellent yields and a simple experimental procedure. The catalyst could be recycled and reused up to three times without any noticeable decrease in the catalytic activity.
\end{abstract}

Keywords: Brønsted acid ionic liquid; $\beta$-indolylketones; Michael addition

\section{Introduction}

$\beta$-indolylketones have received much attention as important building blocks for the synthesis of various natural products and biologically active compounds [1]. Consequently, numerous methods have been developed for their synthesis using various catalysts such as $\mathrm{Bi}(\mathrm{OTf})_{3}$ [2], $\mathrm{HfCl}_{4}$ and $\mathrm{ScCl}_{3}$ [3], $\mathrm{NO}^{+} \mathrm{BF}_{4}^{-}$[4], CAN [5], $\mathrm{InBr}_{3}$ [6], PTSA [7], $\mathrm{Bi}\left(\mathrm{NO}_{3}\right)_{3}$ [8], $\mathrm{GaCl}_{3}$ [9], PVSA [10], $\mathrm{Cu}(\mathrm{OTf})_{2}$ [11], $\mathrm{I}_{2}$ [12], $\mathrm{GaI}_{3}$ [13], PDA [14], FAP [15], $\mathrm{Zr}\left(\mathrm{O}^{\mathrm{t}} \mathrm{Bu}\right)_{4}$ [16] and so on. However, most of the catalysts cannot be recovered and reused because they decompose under the quenching conditions. Furthermore, many of these reported protocols suffer from drawbacks such as strong acidic conditions, long reaction times and low yields of products. Recently, Gu et al. [17] reported silica-supported sodium sulfonate with ionic liquid as catalyst for Michael reactions of indoles. Although the yield was excellent, the reaction temperature is low and the catalyst can be recovered twice, the reaction time is too long. 
Therefore, to avoid these limitations, the introduction of a more efficient method affording higher yields is needed.

In recent years, ionic liquids (ILs) have attracted increasing interest in the context of Green Chemistry owing to their advantageous properties such as negligible vapor pressure, high chemical stability, etc. ILs have gone far beyond the role of solvent, showing their significant importance as new catalysts in controlling the reactions in which they participate [18,19]. Recently, the synthesis of "task-specific" ILs with special functions according to the requirement of a particular reaction has become an attractive field [20,21]. To the best of our knowledge, there have been no reports on the use of Brønsted acid ionic liquids as catalysts for Michael additions of indoles. Herein we wish to report for the first time a novel, environment-friendly and efficient methodology for the synthesis of $\beta$ indolylketones by the reaction of indoles and $\alpha, \beta$-unsaturated ketones using the $\mathrm{SO}_{3} \mathrm{H}$-functionalized Brønsted acid ionic liquid $\left[\mathrm{PyN}\left(\mathrm{CH}_{2}\right)_{4} \mathrm{SO}_{3} \mathrm{H}\right]\left[p-\mathrm{CH}_{3} \mathrm{PhSO}_{3}\right]$ as catalyst (Scheme 1).

Scheme 1. The synthesis of $\beta$-indolylketones catalyzed by $\left[\mathrm{PyN}\left(\mathrm{CH}_{2}\right)_{4} \mathrm{SO}_{3} \mathrm{H}\right]\left[p-\mathrm{CH}_{3} \mathrm{PhSO}_{3}\right]$.<smiles>[R3]C=CC([R4])=O</smiles>

1

2

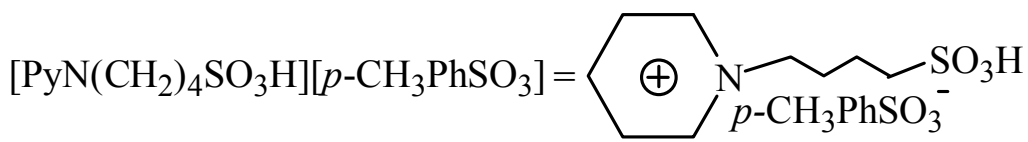

\section{Results and Discussion}

To evaluate the effect of the catalyst $\left[\mathrm{PyN}\left(\mathrm{CH}_{2}\right)_{4} \mathrm{SO}_{3} \mathrm{H}\right]\left[p-\mathrm{CH}_{3} \mathrm{PhSO}_{3}\right]$ under different reaction conditions, the reaction of indole and chalcone was selected as a model reaction. The results are presented in Table 1. Initially the effect of solvent on the reaction was studied (Table 1, entries 1-4) and acetonitrile was found to be the best one. Next, we optimized the amount of $\left[\mathrm{PyN}\left(\mathrm{CH}_{2}\right)_{4} \mathrm{SO}_{3} \mathrm{H}\right][p$ $\mathrm{CH}_{3} \mathrm{PhSO}_{3}$ ] required (Table 1, entries 4-8) and the optimum amount was found to be $10 \mathrm{~mol} \%$. In the absence of $\left[\mathrm{PyN}\left(\mathrm{CH}_{2}\right)_{4} \mathrm{SO}_{3} \mathrm{H}\right]\left[p-\mathrm{CH}_{3} \mathrm{PhSO}_{3}\right]$, the reaction did not yield the desired product (Table 1, entry 5). The influence of the reaction time on the yield was also investigated (Table 1, entries 4, 914). It was clear that the highest yield was produced when the reaction time was $6 \mathrm{~h}$, although the yield did not improve to any greater extent when the reaction time was increased from $4 \mathrm{~h}$ to $6 \mathrm{~h}$. For the purpose of saving energy, we therefore chose $4 \mathrm{~h}$ as the reaction time. Hence, the best condition employs 0.1:1:1 mole ratio of $\left[\mathrm{PyN}\left(\mathrm{CH}_{2}\right)_{4} \mathrm{SO}_{3} \mathrm{H}\right]\left[p-\mathrm{CH}_{3} \mathrm{PhSO}_{3}\right]$, indole and chalcone at $80{ }^{\circ} \mathrm{C}$ for $4 \mathrm{~h}$ using acetonitrile as solvent. 
When optimizing the reaction conditions, the recycling of the catalyst in the reaction of indole and chalcone was investigated. After the separation of products, ethyl acetate was added to the filtrate, which split into two layers. The upper ethyl acetate layer contained unreacted raw material. The lower aqueous layer included the ionic liquid. The lower layer was concentrated on a rotary evaporator at $80{ }^{\circ} \mathrm{C}$ for $1 \mathrm{~h}$ under vacuum to remove excess solvent. [ $\left.\mathrm{PyN}\left(\mathrm{CH}_{2}\right)_{4} \mathrm{SO}_{3} \mathrm{H}\right]\left[p-\mathrm{CH}_{3} \mathrm{PhSO}_{3}\right]$ could be recovered easily and directly reused in subsequent runs. As shown in Table 1, the desired product was obtained in 97, 93, 90\% yields after 1-3 runs, respectively (entry $12^{\mathrm{b}}$ ). This indicated that the ionic liquid $\left[\mathrm{PyN}\left(\mathrm{CH}_{2}\right)_{4} \mathrm{SO}_{3} \mathrm{H}\right]\left[p-\mathrm{CH}_{3} \mathrm{PhSO}_{3}\right]$ was an efficient and recyclable catalyst for the conjugated addtion of indoles to $\alpha, \beta$-unsaturated ketones.

Table 1. Effect of the catalyst $\left[\mathrm{PyN}\left(\mathrm{CH}_{2}\right)_{4} \mathrm{SO}_{3} \mathrm{H}\right]\left[p-\mathrm{CH}_{3} \mathrm{PhSO}_{3}\right]$ under different conditions for the reaction of indole and chalcone.

\begin{tabular}{llccc}
\hline Entry & Solvent & Catalyst (mol \%) & Time (h) & Yield (\%) $^{\mathbf{a}}$ \\
\hline 1 & EtOH & 10 & 6 & 93 \\
2 & Ethyl acetate & 10 & 6 & 88 \\
3 & THF & 10 & 6 & 77 \\
4 & Acetonitrile & 10 & 6 & 98 \\
5 & Acetonitrile & none & 6 & 0 \\
6 & Acetonitrile & 1 & 6 & 74 \\
7 & Acetonitrile & 5 & 6 & 92 \\
8 & Acetonitrile & 15 & 6 & 95 \\
9 & Acetonitrile & 10 & 1 & 79 \\
10 & Acetonitrile & 10 & 2 & 94 \\
11 & Acetonitrile & 10 & 3 & 95 \\
$12^{\mathrm{b}}$ & Acetonitrile & 10 & 4 & $97,93,90$ \\
13 & Acetonitrile & 10 & 5 & 97 \\
14 & Acetonitrile & 10 & 7 & 95 \\
\hline
\end{tabular}

Reaction conditions: indole $(2 \mathrm{mmol})$, chalcone $(2 \mathrm{mmol})$ and catalyst in solvent $(10 \mathrm{~mL}), 80^{\circ} \mathrm{C}$;

${ }^{\mathrm{a}}$ Isolated yield; ${ }^{\mathrm{b}}$ Catalyst was recycled three times.

To explore the generality and scope of this method, a wide variety of substituted indoles and $\alpha, \beta$ unsaturated ketones were reacted with $10 \mathrm{~mol} \%$ of $\left[\mathrm{PyN}\left(\mathrm{CH}_{2}\right)_{4} \mathrm{SO}_{3} \mathrm{H}\right]\left[p-\mathrm{CH}_{3} \mathrm{PhSO}_{3}\right]$ catalyst under the optimized experimental conditions to afford the corresponding $\beta$-indolylketones in excellent yields. (Table 2). The results obtained indicated that the electron donating or withdrawing groups at the indole ring did not seem to affect the reaction significantly in terms of yields, nor do aromatic rings of $\alpha, \beta$ unsaturated ketones.

A plausible mechanism for the conjugate addition of indoles to chalcones is proposed in Scheme 2. $\left[\mathrm{PyN}\left(\mathrm{CH}_{2}\right)_{4} \mathrm{SO}_{3} \mathrm{H}\right]\left[p-\mathrm{CH}_{3} \mathrm{PhSO}_{3}\right]$ coordinates with oxygen atom of $\alpha, \beta$-unsaturated ketones (2) to give intermediate 4. The electron rich $\beta$-position of the indole ring then attacks the electron deficient conjugated carbon-carbon double bond of $\mathbf{4}$ to afford 5 , followed by a hydrogen transfer to yield 6 . Finally 6 rearranged to give target compound 3 and $\left[\mathrm{PyN}\left(\mathrm{CH}_{2}\right)_{4} \mathrm{SO}_{3} \mathrm{H}\right]\left[p-\mathrm{CH}_{3} \mathrm{PhSO}_{3}\right]$ catalyzes the next cycle. 
Table 2. $\left[\mathrm{PyN}\left(\mathrm{CH}_{2}\right)_{4} \mathrm{SO}_{3} \mathrm{H}\right]\left[p-\mathrm{CH}_{3} \mathrm{PhSO}_{3}\right]$-catalyzed synthesis of $\beta$-indolylketones.

\begin{tabular}{|c|c|c|c|c|c|c|c|c|}
\hline \multirow{2}{*}{ Entry } & \multirow{2}{*}{$\mathbf{R}$} & \multirow{2}{*}{$\mathbf{R}_{1}$} & \multirow{2}{*}{$\mathbf{R}_{2}$} & \multirow{2}{*}{$\mathbf{R}_{3}$} & \multirow{2}{*}{$\mathbf{R}_{4}$} & \multirow{2}{*}{ Yields $^{\mathrm{a}}(\%)$} & \multicolumn{2}{|c|}{$\mathrm{Mp}\left({ }^{\circ} \mathrm{C}\right)^{\mathrm{b}}$} \\
\hline & & & & & & & Found & Reported (Lit.) \\
\hline $3 a$ & $\mathrm{H}$ & $\mathrm{H}$ & $\mathrm{H}$ & $\mathrm{Ph}$ & $\mathrm{Ph}$ & 97 & $126-129$ & $127-128[17]$ \\
\hline $3 b$ & $\mathrm{H}$ & $\mathrm{H}$ & $\mathrm{H}$ & $\mathrm{Ph}$ & 4-Cl-Ph & 96 & $153-156$ & $151-152[9]$ \\
\hline $3 \mathrm{c}$ & $\mathrm{H}$ & $\mathrm{H}$ & $\mathrm{H}$ & $\mathrm{Ph}$ & 4- $\mathrm{CH}_{3} \mathrm{O}-\mathrm{Ph}$ & 98 & $174-177$ & \\
\hline $3 d$ & $\mathrm{H}$ & $\mathrm{H}$ & $\mathrm{H}$ & 4-Cl-Ph & $\mathrm{Ph}$ & 90 & $118-121$ & \\
\hline $3 e$ & $\mathrm{H}$ & $\mathrm{H}$ & $\mathrm{H}$ & 4-Cl-Ph & $4-\mathrm{CH}_{3} \mathrm{O}-\mathrm{Ph}$ & 90 & $202-205$ & \\
\hline $3 f$ & $\mathrm{H}$ & $\mathrm{H}$ & $\mathrm{H}$ & $4-\mathrm{CH}_{3}-\mathrm{Ph}$ & $\mathrm{Ph}$ & 98 & $132-134$ & 130-131[9] \\
\hline $3 g$ & $\mathrm{H}$ & $\mathrm{H}$ & $\mathrm{H}$ & $4-\mathrm{CH}_{3}-\mathrm{Ph}$ & 4-Cl-Ph & 94 & $146-149$ & \\
\hline $3 \mathrm{~h}$ & $\mathrm{H}$ & $\mathrm{H}$ & $\mathrm{H}$ & 4- $\mathrm{CH}_{3}-\mathrm{Ph}$ & 4- $\mathrm{CH}_{3} \mathrm{O}-\mathrm{Ph}$ & 96 & $183-186$ & \\
\hline $3 \mathrm{i}$ & $\mathrm{H}$ & $\mathrm{Me}$ & $\mathrm{H}$ & 4-Cl-Ph & 4- $\mathrm{CH}_{3} \mathrm{O}-\mathrm{Ph}$ & 95 & $169-172$ & \\
\hline $3 \mathrm{j}$ & $\mathrm{H}$ & $\mathrm{Me}$ & $\mathrm{H}$ & $4-\mathrm{CH}_{3}-\mathrm{Ph}$ & $4-\mathrm{CH}_{3} \mathrm{O}-\mathrm{Ph}$ & 98 & $172-175$ & \\
\hline $3 \mathrm{k}$ & $\mathrm{H}$ & $\mathrm{H}$ & $5-\mathrm{Br}$ & $\mathrm{Ph}$ & 4-Cl-Ph & 98 & 189-191 & $188-189[13]$ \\
\hline $3 \mathrm{~m}$ & $\mathrm{H}$ & $\mathrm{H}$ & $5-\mathrm{CH}_{3} \mathrm{O}$ & $\mathrm{Ph}$ & $\mathrm{Ph}$ & 92 & $150-153$ & \\
\hline
\end{tabular}

Reaction conditions: indole or substituted indoles $(2 \mathrm{mmol}), \alpha, \beta$-unsaturated ketones $(2 \mathrm{mmol})$, $\left[\mathrm{PyN}\left(\mathrm{CH}_{2}\right)_{4} \mathrm{SO}_{3} \mathrm{H}\right]\left[p-\mathrm{CH}_{3} \mathrm{PhSO}_{3}\right](0.02 \mathrm{mmol})$, acetonitrile $(10 \mathrm{~mL}), 80{ }^{\circ} \mathrm{C}, 4 \mathrm{~h}$; ${ }^{\mathrm{a}}$ Isolated yield; ${ }^{\mathrm{b}}$ Melting points were uncorrected.

Scheme 2. The proposed mechanism of synthesizing $\beta$-indolyketones catalyzed by $\left[\mathrm{PyN}\left(\mathrm{CH}_{2}\right)_{4} \mathrm{SO}_{3} \mathrm{H}\right]\left[p-\mathrm{CH}_{3} \mathrm{PhSO}_{3}\right]$.

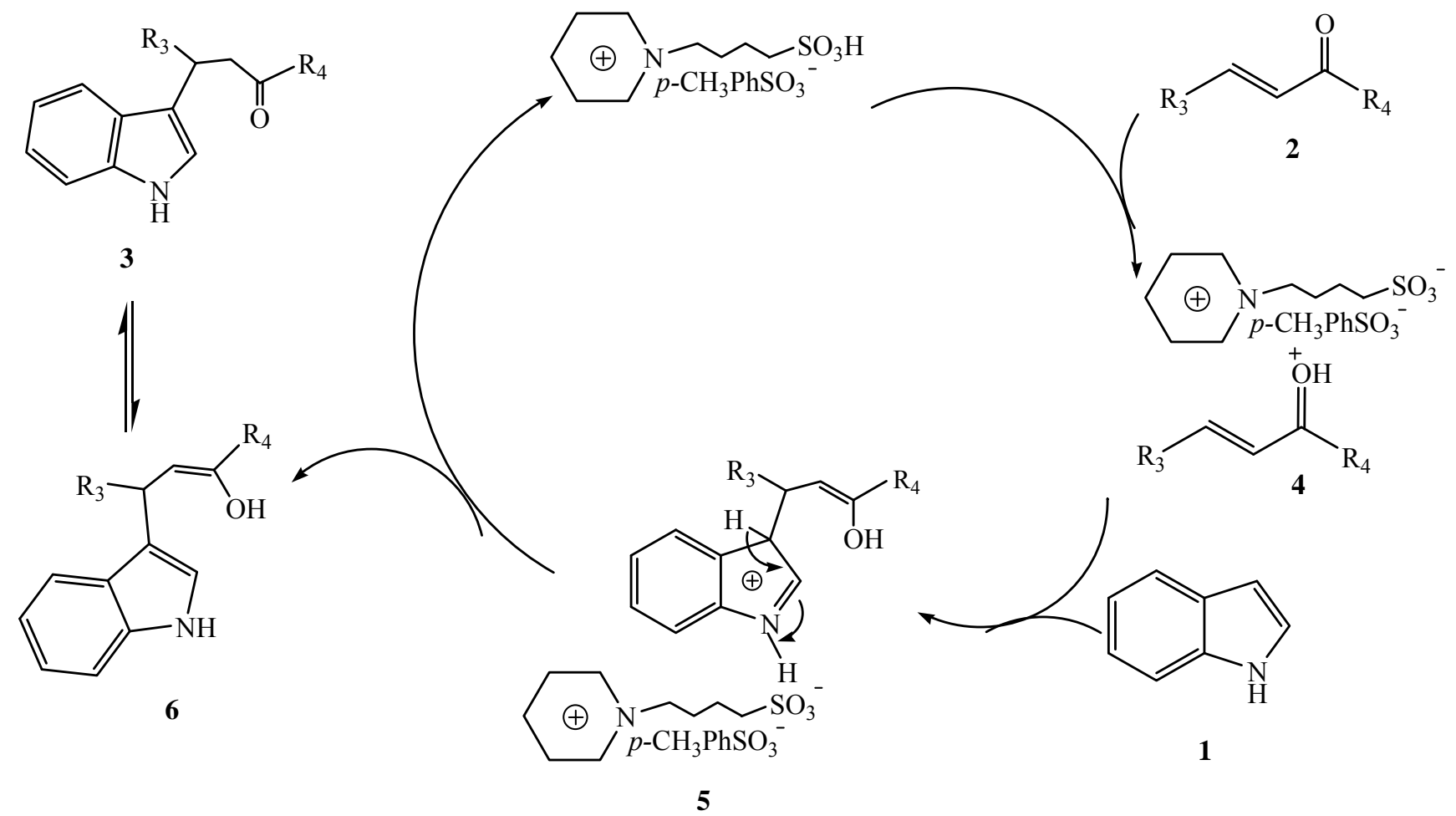




\section{Conclusions}

In conclusion, we have described for the first use of the Brønsted acid ionic liquid $\left[\mathrm{PyN}\left(\mathrm{CH}_{2}\right)_{4} \mathrm{SO}_{3} \mathrm{H}\right]\left[p-\mathrm{CH}_{3} \mathrm{PhSO}_{3}\right]$ as an efficient catalyst for the synthesis of $\beta$-indolylketones. The procedure reported here has the advantages of mild reaction conditions, high yields of products, operational simplicity and catalyst recyclability.

\section{Experimental}

\section{General}

All compounds were characterized by IR, ${ }^{1} \mathrm{H}-\mathrm{NMR}$ spectra and elemental analysis. The IR spectra were obtained as potassium bromide pellets with a FTS-40 spectrometer (BIO-RAD, U.S.A). The ${ }^{1} \mathrm{H}-\mathrm{NMR}$ spectra were obtained on a Varian Inova-400 spectrometer using DMSO- $\mathrm{d}_{6}$ as solvent and TMS as an internal standard; chemical shifts are given in ppm. Elemental analyses $(\mathrm{C}, \mathrm{H}, \mathrm{N})$ were performed on a Perkin-Elmer Analyzer 2400. Melting points were determined using a Büchi B-540 instrument. All melting points are uncorrected. The Brønsted acid ionic liquid $\left[\mathrm{PyN}\left(\mathrm{CH}_{2}\right)_{4} \mathrm{SO}_{3} \mathrm{H}\right][p$ $\mathrm{CH}_{3} \mathrm{PhSO}_{3}$ ] was synthesized according to previous literature [22].

\section{General procedure for the synthesis of $\beta$-indolylketones}

A mixture of indole or substituted indole $(2 \mathrm{mmol}), \alpha, \beta$-unsaturated ketone $(2 \mathrm{mmol})$ and $\left[\mathrm{PyN}\left(\mathrm{CH}_{2}\right)_{4} \mathrm{SO}_{3} \mathrm{H}\right]\left[\mathrm{p}-\mathrm{CH}_{3} \mathrm{PhSO}_{3}\right](0.2 \mathrm{mmol})$ was refluxed at $80{ }^{\circ} \mathrm{C}$ in acetonitrile $(10 \mathrm{~mL})$ for $4 \mathrm{~h}$ with stirring. The completion of the reaction was monitored by TLC. After cooling, the reaction mixture was poured onto crushed ice $(30 \mathrm{~g})$. The resulting precipitate was filtered under suction, and then recrystallized from ethanol to afford the pure product. The results are summarized in Tables 2. All products (except $\mathbf{3 e}, \mathbf{3 g}, \mathbf{3 i}, \mathbf{3 j}, \mathbf{3 m}$ ) are known compounds, which were characterized by mp, IR, ${ }^{1} \mathrm{H}-\mathrm{NMR}$ spectra, elemental analyses.

3-(4-Chlorophenyl)-3-(1H-indol-3-yl)-1-(4-methoxylphenyl)propan-1-one (3e): light yellow powder. Mp 202-205 ${ }^{\circ} \mathrm{C} ;{ }^{1} \mathrm{H}-\mathrm{NMR} \delta: 3.74(\mathrm{dd}, 1 \mathrm{H}, J=7.6,8.0 \mathrm{~Hz}, \mathrm{CH}), 3.83\left(\mathrm{~s}, 3 \mathrm{H}, \mathrm{OCH}_{3}\right), 3.86(\mathrm{dd}, 1 \mathrm{H}$, $J=7.6,8.0 \mathrm{~Hz}, \mathrm{CH}), 4.85$ (t, 1H, $J=7.6 \mathrm{~Hz}, \mathrm{CH}), 6.88-8.00(\mathrm{~m}, 13 \mathrm{H}, \mathrm{ArH}), 10.83$ (s, 1H, NH); IR $\left(v_{\max } ; \mathrm{cm}^{-1}\right): 3383,2971,1657,1599,1489,1256,1180,742$; Anal. calcd. (\%) for $\mathrm{C}_{24} \mathrm{H}_{20} \mathrm{NO}_{2} \mathrm{Cl}: \mathrm{C}$ 73.94, H 5.17, N 3.59. Found: C 73.79, H 5.13, N 3.67.

1-(4-Chlorophenyl)-3-(1H-indol-3-yl)-3-(4-methylphenyl)propan-1-one (3g): light yellow powder. Mp 146-149 ${ }^{\circ} \mathrm{C}$; ${ }^{1} \mathrm{H}-\mathrm{NMR} \delta: 2.19\left(\mathrm{~m}, 3 \mathrm{H}, \mathrm{CH}_{3}\right), 3.77(\mathrm{dd}, 1 \mathrm{H}, J=7.6,7.6 \mathrm{~Hz}, \mathrm{CH}), 3.88(\mathrm{dd}, 1 \mathrm{H}, J=7.6$, $7.6 \mathrm{~Hz}, \mathrm{CH}), 4.80(\mathrm{t}, 1 \mathrm{H}, J=7.6 \mathrm{~Hz}, \mathrm{CH}), 6.85-8.03(\mathrm{~m}, 13 \mathrm{H}, \mathrm{ArH}), 10.83(\mathrm{~s}, 1 \mathrm{H}, \mathrm{NH})$; IR $\left(v_{\max }\right.$; $\mathrm{cm}^{-1}$ ): 3418, 2914, 1674, 1587, 1455, 1244, 1179, 741; Anal. Calcd. (\%) for $\mathrm{C}_{24} \mathrm{H}_{20} \mathrm{NOCl}$ : C 77.10, $\mathrm{H}$ 5.39, N 3.75. Found C 77.27, H 5.34, N 3.68.

3-(4-Chlorophenyl)-1-(4-methoxylphenyl)-3-(2-methyl-1H-indol-3-yl)propan-1-one(3i): brown powder. Mp 169-172 ${ }^{\circ} \mathrm{C} ;{ }^{1} \mathrm{H}-\mathrm{NMR} \delta: 2.38\left(\mathrm{~s}, 3 \mathrm{H}, \mathrm{CH}_{3}\right) ; 3.81\left(\mathrm{~s}, 3 \mathrm{H}, \mathrm{OCH}_{3}\right) ; 3.87(\mathrm{dd}, 1 \mathrm{H}, J=7.6,7.6 \mathrm{~Hz}$, 
$\mathrm{CH}), 3.90(\mathrm{dd}, 1 \mathrm{H}, J=7.6,7.6 \mathrm{~Hz}, \mathrm{CH}), 4.88(\mathrm{t}, 1 \mathrm{H}, J=7.6 \mathrm{~Hz}, \mathrm{CH}), 6.82-8.19(\mathrm{~m}, 12 \mathrm{H}, \mathrm{ArH}), 10.74$ $(\mathrm{s}, 1 \mathrm{H}, \mathrm{NH})$; IR $\left(v_{\max } ; \mathrm{cm}^{-1}\right): 3312,1664,1596,1464,1258,1169,748$; Anal. Calcd. (\%) for $\mathrm{C}_{25} \mathrm{H}_{22} \mathrm{NO}_{2} \mathrm{Cl}$ : C 74.34, H 5.49, N 3.47. Found C 74.45, H 5.43, N 3.52.

1-(4-Methoxylphenyl)-3-(2-methyl-1H-indol-3-yl)-3-(4-methylphenyl)propan-1-one (3j): light yellow powder. Mp 172-175 ${ }^{\circ} \mathrm{C} ;{ }^{1} \mathrm{H}-\mathrm{NMR} \delta: 2.2\left(\mathrm{~s}, 3 \mathrm{H}, \mathrm{CH}_{3}\right) ; 2.36\left(\mathrm{~s}, 3 \mathrm{H}, \mathrm{CH}_{3}\right) ; 3.80\left(\mathrm{~s}, 3 \mathrm{H}, \mathrm{OCH}_{3}\right) ; 3.84$ (dd, 1H, $J=7.2,7.6 \mathrm{~Hz}, \mathrm{CH}$ ), 3.86 (dd, 1H, $J=7.2,7.6 \mathrm{~Hz}, \mathrm{CH}), 4.85$ (t, 1H, $J=7.6 \mathrm{~Hz}, \mathrm{CH}), 6.81-$ $7.94(\mathrm{~m}, 12 \mathrm{H}, \mathrm{ArH}), 10.68$ (s, 1H, NH); IR $\left(v_{\max } ; \mathrm{cm}^{-1}\right): 3338,1663,1595,1463,1260,1168,747$; Anal. Calcd. (\%) for $\mathrm{C}_{26} \mathrm{H}_{25} \mathrm{NO}_{2}$ : C 81.43, H 6.57, N 3.65. Found C 81.29, H 6.51, N 3.73.

1,3-Diphenyl-3-(5-methoxyl-1H-indol-3-yl)propan-1-one (3m): white powder. Mp 150-153 ${ }^{\circ} \mathrm{C}$; ${ }^{1} \mathrm{H}-\mathrm{NMR} \delta 3.67$ (s, 3H, $\mathrm{OCH}_{3}$ ), 3.80 (dd, 1H, $\left.J=7.2,7.6 \mathrm{~Hz}, \mathrm{CH}\right), 3.90(\mathrm{dd}, 1 \mathrm{H}, J=7.2,7.6 \mathrm{~Hz}, \mathrm{CH})$, $4.82(\mathrm{t}, 1 \mathrm{H}, J=7.2 \mathrm{~Hz}, \mathrm{CH}), 6.66-8.02(\mathrm{~m}, 14 \mathrm{H}, \mathrm{ArH}), 10.68(\mathrm{~s}, 1 \mathrm{H}, \mathrm{NH}) ; \mathrm{IR}\left(v_{\max } ; \mathrm{cm}^{-1}\right): 3365$, 1678, 1597, 1485, 1214, 1169, 723; Anal. Calcd. (\%) for $\mathrm{C}_{24} \mathrm{H}_{21} \mathrm{NO}_{2}: \mathrm{C} 81.10, \mathrm{H}$ 5.96, N 3.94. Found C. 80.93, H 6.01, N 3.90 .

\section{Acknowledgements}

We gratefully ackonwledge support from the National Natural Science Foundation of China (No. 20862016).

\section{References}

1. Zhan, Z.P.; Yang, R.F.; Lang, K. Samarium Triiodide-Catalyzed Conjugate Addition of Indoles With Electron-deficient Olefins. Tetrahedron Lett. 2005, 46, 3859-3862.

2. Reddy, A.V.; Ravinder, K.; Goud, T.V.; Krishnaiah, P.; Raju, T.V.; Venkateswarlu, Y. Bismuth Triflate Catalyzed Conjugate Addition of Indoles to $\alpha$, $\beta$-enones. Tetrahedron Lett. 2003, 44, 6257-6260.

3. Kawatsura, M.; Aburatani, S.; Uenishi, J. Catalytic Conjugate Addition of Heterocyclic Compounds to $\alpha, \beta$-Unsaturated Carbonyl Compounds by Hafnium Salts and Scandium Salts. Tetrahedron 2007, 63, 4172-4177.

4. Wu, G.L.; Wu, L.M. Nitrosonium $\left(\mathrm{NO}^{+}\right)$Catalyzed Michael Addition of Indoles to Unsaturated Enones. Chin. Chem. Lett. 2008, 19, 55-58.

5. Ko, S.K.; Lin, C.C.; Tu, Z.J.; Wang, Y.F.; Wang, C.C.; Yao, C.F. CAN and Iodine-Catalyzed Reaction of Indole or 1-Methylindole with $\alpha, \beta$-Unsaturated Ketone or Aldehyde. Tetrahedron Lett. 2006, 47, 487-492.

6. Bandini, M.; Cozzi, P.G.; Giacomini, M.; Melchiorre, P.; Selva, S.; Umani-Ronchi, A. Sequential One Pot $\mathrm{InBr}_{3}$-Catalyzed 1,4- then 1,2-Nucleophilic Addition to Enones. J. Org. Chem. 2002, 67, 3700-3704.

7. Ji, S.J.; Wang, S.Y. An Expeditious Synthesis of $\beta$-indolylketones Catalyzed by $p$-toluenesulfonic Acid (PTSA) Using Ultrasonic Irradiation. Ultrason. Sonochem. 2005, 12, 339-343.

8. Srivastava, N.; Banik, B.K. Bismuth Nitrate-Catalyzed Versatile Michael Reactions. J. Org. Chem. 2003, 68, 2109-2114. 
9. Xu, R.; Ding, J.C.; Chen, X.A.; Liu, M.C.; Wu, H.Y. Gallium Trichloride-Catalyzed Conjugate Addition of Indole and Pyrrole to $\alpha, \beta$-Unsaturated Ketones in Aqueous Media. Chin. Chem. Lett. 2009, 20, 676-679.

10. Ekbote, S.S.; Panda, A.G.; Bhor, M.D.; Bhanage, B.M. Polyvinylsulfonic Acid as a Novel Brønsted Acid Catalyst for Michael Addition of Indoles to $\alpha, \beta$-Unsaturated Ketones. Catal. Commun. 2009, 10, 1569-1573.

11. Yadav, J.S.; Reddy, B.V.S.; Baishya, G.; Reddy, K.V.; Narsaiah, A.V. Conjugate addition of Indoles to $\alpha, \beta$-unsaturated Ketones Using $\mathrm{Cu}(\mathrm{OTf})_{2}$ Immobilized in Ionic Liquids. Tetrahedron 2005, 61, 9541-9544.

12. Bimal, K.B.; Miguel, F.; Clarissa, A. Iodine-Catalyzed Highly Efficient Michael Reaction of Indoles under Solvent-Free Condition. Tetrahedron Lett. 2005, 46, 2479-2482.

13. Huang, Z.H.; Zou, J.P.; Jiang, W.Q. Gallium(III) Triiodide Catalyzed Conjugate Addition of Indoles with $\alpha$, $\beta$-Unsaturated Ketones. Tetrahedron Lett. 2006, 47, 7965-7968.

14. Zhou, W.; Xu, L.W.; Yang, L.; Zhao, P.Q.; Xia, C.G. Novel Brønsted Acid-Catalyzed Michaeltype Friedel-Crafts Reactions of Indoles and Acetalization of Aldehydes. J. Mol. Catal. A Chem. 2006, 249, 129-134.

15. Tahir, R.; Banert, K.; Sebti, S. Friedel-Crafts-type Conjugate Addition of Indoles Using Fluorapatite Doped Zinc Bromide as Efficient Solid Catalyst. Appl. Catal. A: Gen. 2006, 315, 147-149.

16. Blay, G.; Fernndez, I.; Pedro, J.R.; Vila, C. Highly Enantioselective Friedel-Crafts Alkylations of Indoles with Simple Enones Catalyzed by Zirconium(IV)-BINOL Complexes. Org. Lett. 2007, 9 , 2601-2604.

17. Gu, Y.; Ogawa, C.; Kobayashi, S. Silica-Supported Sodium Sulfonate with Ionic Liquid: A Neutral Catalyst System for Michael Reactions of Indoles in Water. Org. Lett. 2007, 9, 175-178.

18. Welton, T. Room-Temperature Ionic Liquids. Solvents for Synthesis and Catalysis. Chem. Rev. 1999, 99, 2071-2084.

19. Pârvulescu, V.I.; Hardacre, C. Catalysis in Ionic Liquids. Chem. Rev. 2007, 107, 2615-2665.

20. Ranu, B.C.; Banerjee, S. Ionic Liquid as Catalyst and Reaction Medium. The Dramatic Influence of a Task-Specific Ionic Liquid, [bmIm]OH, in Michael Addition of Active Methylene Compounds to Conjugated Ketones,Carboxylic Esters, and Nitriles. Org. Lett. 2005, 7, 3049-3052.

21. Qiao, K.; Hagiwara, H.; Yokoyama, C. Acidic Ionic Liquid Modified Silica Gel as Novel Solid Catalysts for Esterification and Nitration Reactions. J. Mol. Catal. A Chem. 2006, 246, 65-69.

22. Liu, X.; Zhou, J.X.; Guo, X.W.; Liu, M.; Ma, X.1.; Song, C.S.; Wang, C. $\mathrm{SO}_{3} \mathrm{H}-$ Functionalized Ionic Liquids for Selective Alkylation of $p$-Cresol with tert-Butanol. Ind. Eng. Chem. Res. 2008, 47, 5298-5303.

Sample Availability: Available from the authors.

(C) 2009 by the authors; licensee Molecular Diversity Preservation International, Basel, Switzerland. This article is an open-access article distributed under the terms and conditions of the Creative Commons Attribution license (http://creativecommons.org/licenses/by/3.0/). 